\title{
Ambiguity in Leadership Communication: A Latent Hazard in the Aviation Community?
}

\author{
James Bryan Burrows-McElwain ${ }^{1}$, Ibibia Karisemie Dabipi ${ }^{1}$ and Dollie McCormick $^{2}$ \\ 1. University of Maryland Eastern Shore, Princess Anne, MD 21853, USA \\ 2. NOVA Southeastern University, Fort Lauderdale, FL 33314, USA
}

\begin{abstract}
In aviation, it is common to have several aircraft operating in similar conditions uneventfully, yet another aircraft, following suit, experiences an accident in the same conditions. With the same information available to crews of both aircraft, conditions can quickly change leading to an accident for one, while the other crews land safely. Should this situational ambiguity be the cause of the accident/incident instead of the crew's poor judgment in light of the fact that others made the same decision without error? Can industry policies communicated to the crew on how to behave in these ambiguous situations be at fault? Do current practices tolerate this type of ambiguity? This paper explores the concept of ambiguity in leadership communication in the context of the airline industry. The authors define the variable of ambiguity in the context of National Airspace System operations and explore its impact on safety. Ambiguity is viewed as a latent hazard in the cockpit, the air traffic control environment, and operations components of the aviation industry. The concept of ambiguity is defined and explored using aviation case study to highlight its influence on aviation safety and decision making using the Reason Accident Causation Model. Additionally, this paper explores various survey instruments and scales that might be considered for observing and measuring these phenomena in the aviation community and recommends best practices.
\end{abstract}

Key words: Ambiguity, leadership, aviation community, communication.

\section{Introduction}

On November 20th, 2013, an oversized Boeing 747 Dreamlifter landed at McConnell Air Force Base (AFB) in Kansas [1] instead of its originally planned destination. The aircraft operated by Atlas Air left John F. Kennedy Airport in New York City, bound for McConnell AFB but instead landed at a general aviation airport just 12 miles before the airport of intended landing, Col. James Jabara Airport. Not long into the new year, a similar event took place when a Southwest Airlines flight departed Chicago, bound for Branson, Missouri (KBKG) instead landed at Taney County Airport (KPLK), about 8 miles prior to Branson but along the flight path [2]. The flight crews in both scenarios were operating under VFR (visual flight rules) and were making visual approaches to the

Corresponding author: James Bryan Burrows-McElwain, $\mathrm{PhD}$, Management \& Program Analyst, Adjunct Professor, Research fields: Organizational/Human Factors; Aviation Safety; Leadership Theory. airport which means they were not relying on instrumentation for vertical or horizontal guidance/navigation. In both of these cases, ambiguity because of similar airport markings, flight crew decision-making (pilotage) did not result in any injuries, though a latent hazard was exposed. An exploration of the concept of crewmember tolerance to ambiguity and its effect(s) on safety is in order.

\section{Ambiguity and Its Link to Safety Culture}

Ambiguity is defined as, "Doubtfulness or uncertainty as regards interpretation" [3]. Budner [4] first defined intolerance of ambiguity as, "the tendency to perceive ambiguous situations as a source of threat". McLain [5] defined ambiguity as a, "term we apply to perceived insufficiency of information regarding a particular stimulus or context". He argued that researchers must define ambiguity and tolerance separately with tolerance representing begrudging acceptance and ambiguity as the perception of 
inadequate information needed to understand information appropriately.

Ambiguity as a leadership construct was introduced by Lane and Klenke [6] in the context of corporate leadership in an age of information overload during periods of uncertainty. Lane and Klenke proposed that although ambiguity has been linked to leadership theory, a robust evolution of ambiguity into larger leadership frameworks has yet to occur. They noted that ambiguity in the workplace arises in new situations where cues are lacking, situations where cues are overwhelmingly present, and situations where cues are contradictory. Lane and Klenke identified ambiguity as an additional element of McCormick's model of cognitive social theory of leadership confidence. Additionally, researchers [7, 8] have pointed out the impact of technology in leadership effectiveness. The field of aviation is in a constant state of technological change, as the FAA (Federal Aviation Administration). FAA is implementing new emergent technologies, being able to measure an organization's tolerance of ambiguity [9] will be a necessary tool that leaders will need to mitigate potential hazards in our airspace systems.

Ambiguity as it relates to aviation safety has been well documented. A study conducted to review 37 behavior caused accidents identified by the NTSB (National Transportation Research Board) identified ambiguity as a contributing factor [10]. Leaders and decision makers have a myriad of situational elements that constantly change posing a real challenge to one's ability to assess the current state. Orasanu and Martin state that leaders often have to justify their decisions to others. In ambiguous situations, leaders may choose not to act out of fear of a lack of justification for choosing a new course of action. The result is inaction or PCE (plan continuation error) where the original course of action is kept. Orasanu and Martin recommend developing tools to assist leaders in aviation to change existing behaviors, make difficult decisions to avoid incidents and accidents.
In a separate study, Orasanu, Fischer and Davidson [11] investigated uncertainty and risk perception in aviation safety. Orasanu et al. found that while pilots do consider many safety factors in their decision-making processes, organizational variables were not addressed. Researchers stated that, "Difference between pilots in their choices of more or less risk tolerant decisions clearly reflects the ambiguity inherent in the problem cues and uncertainties concerning solution outcomes". That is, researchers could not identify a general agree upon correct interpretation of the scenario. In short, risk assessment by pilots was found to be subjective during ambiguous operating conditions.

Wiegmann and Shappell [12] indicated that ambiguity in safety policy within an organization will cause safety to suffer. Examples include ill-defined, unofficial or conflicting policies and values. Westrum and Adamski [13] argue that leaders with, "high integrity attitudes and behaviors form a coherent pattern". This suggests that moral and ethical leadership of organizational supervisors will result in less ambiguity in the workplace.

\subsection{Case-Study 1: Pinnacle Airlines Flight 4712}

On April 12, 2007, Pinnacle Airlines flight 4712, a Bombardier/ CRJ (Canadair Regional Jet), N8905F, ran off the end of runway 28 after landing at Cherry Capital Airport (KTVC), Traverse City, Michigan. Although sustaining substantial damage, none of the 49 passengers or 3 crewmembers was injured. It was snowing at the time, the flight was operated under IMC (instrument meteorological conditions), and the flight was on an IFR (instrument flight rules) flight plan.

\subsubsection{Probable Cause}

The National Transportation Safety Board determined that the probable cause of this accident was the pilots' decision to land at the airport without performing a landing distance assessment, which may have been because the crew was tired after a long duty 
day. The NTSB determined that the major contributions to the accident included (1) the existing Federal Aviation Administration pilot flight and duty time regulations that permitted the pilots' long, demanding duty day and (2) the airport operations supervisor's use of ambiguous and unspecific radio phraseology in providing runway braking information.

\subsubsection{NTSB Recommendations}

As a result of this investigation, the National Transportation Safety Board made the following recommendation related to ambiguity in communications to the Federal Aviation Administration [14]:

...emphasizes the importance of specific and decisive radio communications, and urges airports to ensure that those criteria are being met in all airfield radio communications (A-08-42).

The findings highlight both individual and organizational communication issues that are organizational in nature.

\subsection{Case Study II: Gulfstream Aerospace Corp}

On April 2, 2011, about 0934 mountain daylight time, an experimental Gulfstream Aerospace Corporation GVI (G650), N652GD, crashed during takeoff from runway 21 at Roswell International Air Center Airport, Roswell, New Mexico. The two pilots and the two flight test engineers were fatally injured, and the airplane was substantially damaged by impact forces and a post-crash fire. The airplane was registered to and operated by Gulfstream as part of its G650 flight test program. The flight was conducted under the provisions of 14 Code of Federal Regulations Part 91. Visual meteorological conditions prevailed at the time of the accident.

The accident occurred during a planned OEI (one-engine-inoperative) takeoff exercise when a stall on the right outboard wing produced a rolling moment that the flight crew was not able to control. This led to the right side wingtip contacting the runway and the airplane departing the runway from the right side. The aircraft impacted a hardened concrete structure and an airport weather station, which caused structural damage and a fire that consumed the Gulfstream.

The NTSB's (National Transportation Safety Board) investigation of this accident found that the airplane stalled while lifting off the ground. As a result, the NTSB examined the role of "ground effect" on the airplane's performance. Ground effect refers to changes in the airflow over the airplane resulting from the proximity of the airplane to the ground. Ground effect results in increased lift and reduced drag at a given AOA (angle of attack) as well as a reduction in the stall AOA. In preparing for the G650 field performance flight tests, Gulfstream considered ground effect when predicting the airplane's takeoff performance capability but overestimated the in ground effect stall AOA. Consequently, the airplane's AOA threshold for stick shaker (stall warning) activation and the corresponding pitch limit indicator (on the primary flight display) were set too high, and the flight crew received no tactile or visual warning before the actual stall occurred.

\subsubsection{Conclusions}

(1) Given the airplane's low altitude, the time-critical nature of the situation, and the ambiguous stall cues presented in the cockpit, the flight crew's response to the stall event was understandable;

(2) Because Gulfstream did not clearly define the roles and responsibilities for on-site test team members, critical safety-related parameters were not being adequately monitored and test results were not being sufficiently examined during flight testing on the day of the accident;

(3) External safety audits would help Gulfstream monitor the implementation of safety management principles and practices into its flight test operations and sustain long-term cultural change.

\subsubsection{Probable Cause}

The National Transportation Safety Board determined that the cause of the accident was an aerodynamic stall and subsequent uncommanded roll 
during a one engine-inoperative takeoff flight test. The roll and stall were the result of (1) the Gulfstream's failure to properly develop and validate takeoff speeds for the flight tests and recognize and correct the takeoff safety speed (V2) error during previous flight tests, (2) the flight test team's attempts to achieve V2 speeds that were erroneously low, and (3) the company's inadequate investigation of previous G650 uncommanded roll events, which indicated that the company's estimated stall angle of attack while the airplane was in ground effect was too high. Contributing to the accident was the organization's failure to effectively manage the G650 flight test program roles and responsibilities. The NTSB highlighted a need for more oversight of engineering processes to ensure they received sufficient oversight, ensuring that potential hazards were identified, a risk controls had been put in place.

\subsubsection{Recommendation}

As a result of the accident, the NTSB made the following recommendations to the Gulfstream Aerospace Corporation regarding their flight testing in regards to safety management:

(1) Commission an audit by qualified independent safety experts, before the start of the next major certification flight test program, to evaluate the company's flight test safety management system, with special attention given to the areas of weakness identified in this report, and address all areas of concern identified by the audit;

(2) Provide information about the lessons learned from the implementation of its flight test safety management system to interested manufacturers, flight test industry groups, and other appropriate parties.

\section{Ambiguity, Safety Climate and Authentic Leadership}

Leadership and organizational culture have been intimately linked in previous research. In aviation organizations, leadership is responsible for safety and can have an impact on the climate of that organization. James Reason's [15] Accident Causation Model identifies an organization's leadership as the first layer of defense available to an organization to mitigate a potential accident. Due to the relatively low accident rate among commercial operations in the United States NAS (National Airspace System), it is difficult to identify a significant and sensitive predictor [16]. A predictive model of aviation safety culture was constructed using multiple regression analysis to identify independent predictor variables. A hierarchical regression analysis was conducted to test predictor variables identified in the literature. Independent variables identified in the literature included authentic leadership and lack of tolerance of ambiguity as potential building blocks. Populations of 119 aviation professionals, from varying disciplines, were surveyed using an online survey tool. Of particular interest, the impact of a key leadership variable, leader authenticity was tested. Variables correlated well to Safety climate. Ambiguity moderately correlated (Table 1) positively to Safety Climate $(\mathrm{r}=.312)$; Leader Authenticity had a strong positive correlation to Safety Climate $(\mathrm{r}=.664)$. When leadership authenticity was added to the model, the model accounted for almost half of the variability (48.3\%). The coefficient of multiple determination (R2) change was a $35.1 \%$ increase in predictive capacity of the model after adding the leader authenticity variable. That $\mathrm{R} 2$ change was associated with an $\mathrm{F}$ value of 80.26 with one degree of freedom with 115 in the denominator, the $\mathrm{F}$ change associated with the R2 change is statistically significant to the .0001 level. This demonstrated that adding leadership authenticity to the model increases the model's predictive capacity in predicting overall safety climate in a statistically

Table 1 Two-tailed test of significance

\begin{tabular}{llll}
\hline Measure & 1 & 2 & 3 \\
\hline Safety climate & --- & & \\
Tol. for ambiguity & $r=0.312 * * *$ & --- \\
Authenticity & $r=0.664 * * *$ & $r=0.181 *$ & --- \\
\hline$* * * p<0.001 ;^{*} p<0.05 .($ Example $r=0.312, \mathrm{n}=119, p<0.001)$
\end{tabular}




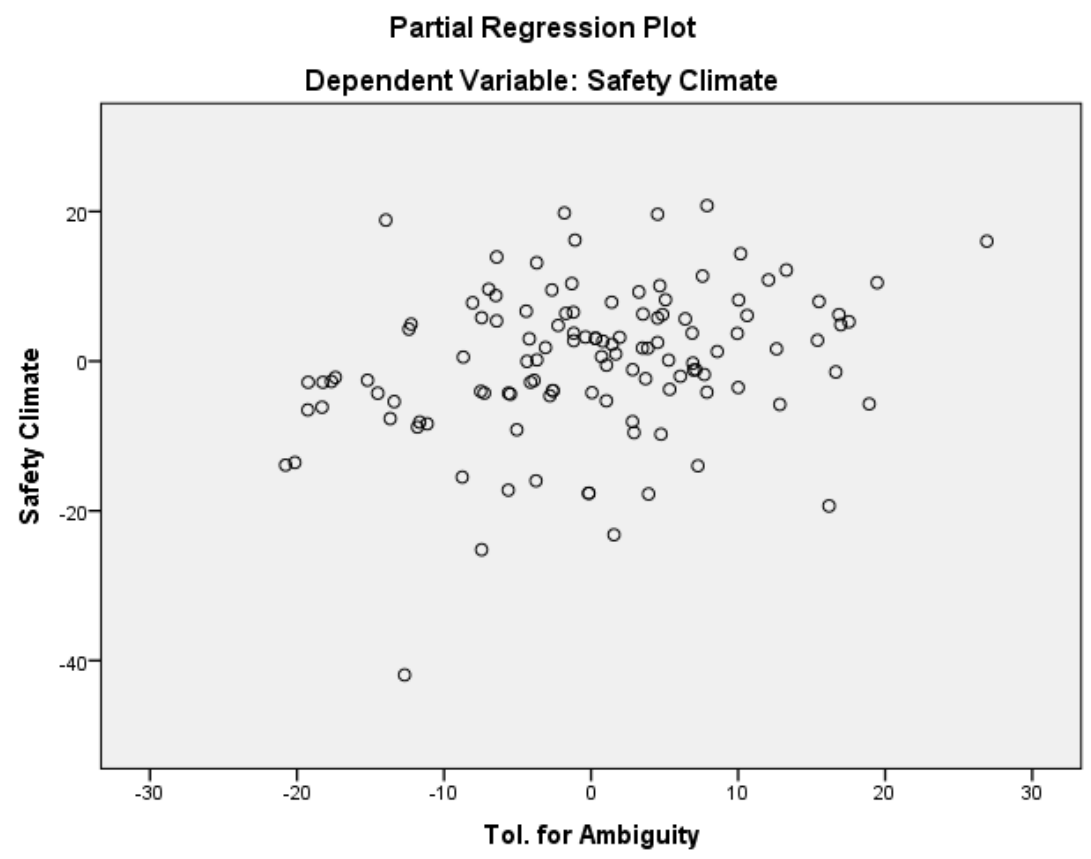

Fig. 1 Scatter plot: safety climate \& tolerance for ambiguity.

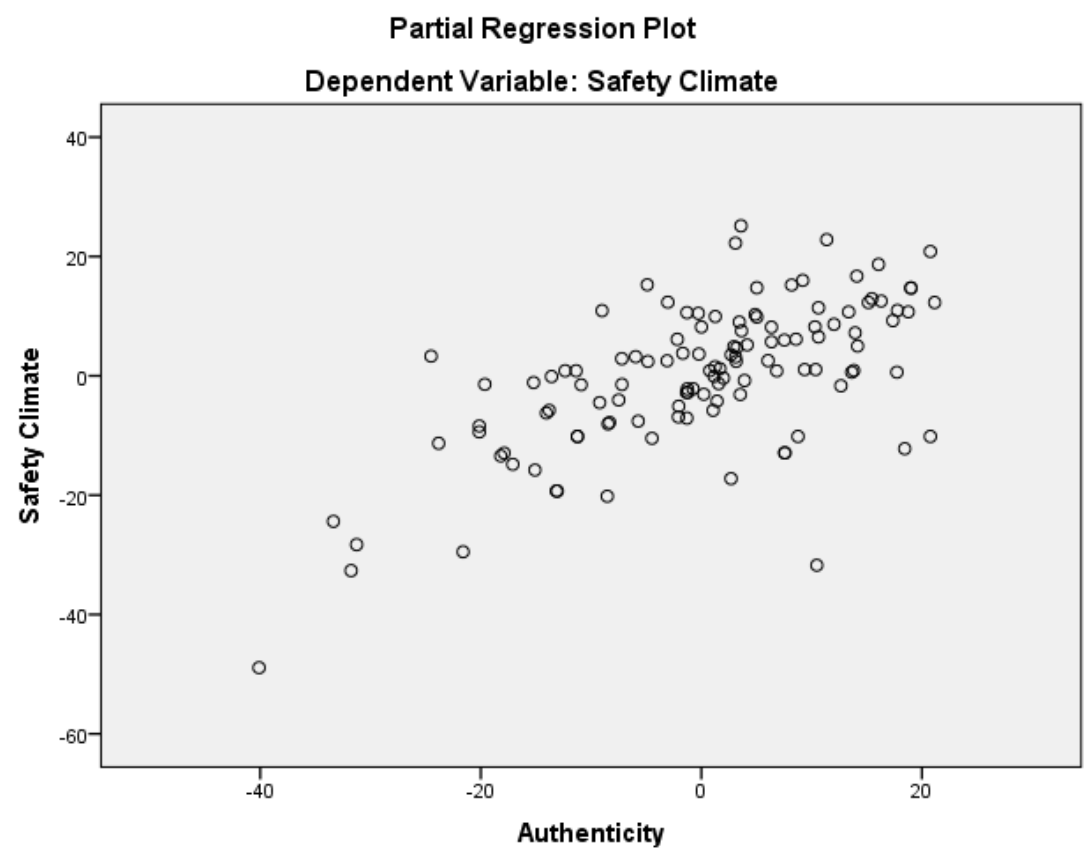

Fig. 2 Scatter plot: safety climate and leader authenticity.

significant way and increases the percentage of variance counted for by $35.1 \%$ [9].

\section{Conclusions}

Leader authenticity and Tolerance for ambiguity proved to be significant indicators of safety climate and in terms of individual relationships between the independent variables and safety climate, leader authenticity $(\mathrm{t}=8.959, \mathrm{p}<0.001)$ and tolerance for ambiguity $(\mathrm{t}=3.218, \mathrm{p}<0.001)$. 
In aviation organizations where safety risks have high consequences, ambiguity is less tolerated. Due to the relatively low numbers of aviation accidents, it has proven more challenging to identify significant and sensitive predictors [16] of latent hazards that might trigger future accidents. James Reason [15] indicated that the first layer of defense for an organization in mitigating a debilitating hazard, incident or accident is the leadership in the organization. The results of the multiple regression analysis indicated that authentic leadership, which embraces balanced processing, and tolerance for ambiguity have influence on overall organizational safety climate and may be an additional tool practitioners can utilize in predicting overall safety climate for operators. This may be due to the increase in communication and collaboration adopted by authentic leaders who aim to balance their decision making with external feedback. Additionally, sharing information (transparency) and using that feedback to gain a better appreciation of the actual state of affairs (self-awareness) may contribute to the employee's perception(s) and overall climate of safety.

Continued research with larger and more diverse aviation industry populations are worth investigation. As sub-cultures may be present, independent variables may have greater or lesser impact on differing aviation sub-groups (air traffic, technical operations, airline flight crew; ground operations; maintenance and repair, etc.). Additionally, other independent variables such as demographics and situational leadership elements may play a role in the maintenance of an organization's safety climate.

\section{References}

[1] CNN. 2013. "Cargo Jet Takes off from Wichita on Short Runway." Accessed on January 1st, 2014. http://www.cnn.com/2013/11/21/travel/kansas-cargo-plan e-wrong-airport/

[2] Forbes. 2014. "Southwest Airlines Plane Lands at Wrong Airport, Almost Careens Off Cliff." Retrieved on January 15, 2014. http://www.forbes.com/sites/grantmartin/2014/ 01/12/southwest-airlines-plane.

[3] Free Dictionary. 2014. "Definition of Ambiguity." Retrieved on January 1, 2014. http://www. thefreedictionary.com/ambiguity.

[4] Budner, S. 1962. "Tolerance of Ambiguity as a Personality Variable." Journal of Personality 30: 29-40.

[5] McLain, D. 1993. "The MSTAT-I: A New Measure of an Individual's Tolerance for Ambiguity." Educational and Psychological Measurement 53: 183-9.

[6] Lane, M., and Klenke, K. 2004. "The Ambiguity Tolerance Interface: A Modified Social Cognitive Model for Leading under Uncertainty." Journal of Leadership \& Organizational Studies 10 (3): 69-81.

[7] Hendrick, H. 1991. "Ergonomics in Organizational Design and Management.” Ergonomics 34 (6): 743-56.

[8] Klenke, K. 1992. "Leadership Processes in Computer Mediated Work Groups: Implications of Information Systems Development and Leadership Studies."

[9] Burrows-McElwain. 2015. "Leader Authenticity, Tolerance for Ambiguity and Burnout: Some Predictors of Positive Safety Climate in Aviation." Doctoral dissertation, Frederick Douglas Library, University of Maryland Eastern Shore.

[10] Orasanu, J., and Martin, L. 1998. "Errors in Aviation Decision Making: A Factor in Accidents and Incidents." In 2nd Workshop on Human Error, Safety, and System Development, 100-7.

[11] Orasanu, J., Fischer, U., and Davison, J. 2002. "Risk Perception: A Critical Element of Aviation Safety." In 15th Triennial World Congress.

[12] Wiegmann, D., and Shappell, S. 2001. "Human Error Analysis of Commercial Aviation Accidents: Application of the HFACS (Human Factors Analysis and Classification System)." Aviation, Space, and Environmental Medicine 22 (11): 1006-16.

[13] Westrum, R., and Adamski, A. 1999. Handbook of Aviation Human Factors: Organizational factors Associated with Safety and Mission Success in Aviation Environments, 67-103.

[14] NTSB. 2012. Aircraft Accident Report Numbers AAR-08-02 \& AAR-12/02. http://www.ntsb. gov/investigations/summary/AAR0802.html \& http://www.ntsb.gov/news/events/2012/gulfstream/.

[15] Reason, J. 1995. "A Systems Approach to Organizational Error.” Ergonomics 38 (8): 1708-21.

[16] O'Connor, P., O'Dea, A., Kennedy, Q., and Buttrey, S. E. 2011. "Measuring safety climate in aviation: A review and recommendations for the future." Safety Science 49 (2): 128-38. 